\title{
Extended Spatio-temporal Relations between Moving and Non-moving Objects
}

\author{
Chang Choi ${ }^{1}$, Junho Choi ${ }^{1}$, Juhyun Shin ${ }^{1}$, \\ Ilsun $\mathrm{You}^{2}$, and Pankoo Kim ${ }^{3, *}$ \\ ${ }^{1}$ Dept. of Computer Engineering, Chosun University, \\ 375 Seosuk-dong Donggu, Gwangju 501-759, South Korea \\ enduranceaura@gmail.com, xdman@paran.com, \\ jhshinechosun.ac.kr \\ ${ }^{2}$ School of Information Science, Korean Bible University, \\ 16 Danghyun 2-gil, Nowon-gu, Seoul 139-791, South Korea \\ isyou@bible.ac. kr \\ ${ }^{3}$ Dept. of CE, Chosun University, South Korea \\ pkkim@chosun.ac.kr
}

\begin{abstract}
During the last decade, the emerging technology for video retrieval is mainly based on the content. However, semantic-based video retrieval has become more and more necessary for the humans especially the naive users who can only use the human language during retrieval. In this paper, we focus on semantic representation using topological and directional relations between non-moving and moving objects for security using CCTV(closed-circuit Television). In this paper, we propose new spatio-temporal relation to extend previous work using topological and directional relations and investigate spatiotemporal predicates which propose our models. In the experiment part, we compared retrieval results using TSR(Tangent Space Representation) with those using rules represented by the proposed model.
\end{abstract}

Keywords: Spatio-temporal Relation, Motion Recognition, Motion Predicates.

\section{Introduction}

During the last decade, the emerging technology for video retrieval is mainly based on the content. However, semantic-based video retrieval has become more and more necessary for the humans especially the naive users who can only use the human language during retrieval. So, semantic-based video retrieval research has caused many researchers' attentions.

Since the most important semantic information for video is based on video motion research which is the significant factor for video event representation, there has been a significant amount of event understanding research in various application domains[12]. One major goal of this research is to accomplish the automatic extraction of feature semantics from a motion and to provide support for

\footnotetext{
* Corresponding author.
} 
semantic-based motion indexing, retrieval and management. Most of the current approaches to activity recognition are composed of defining models for specific activity types that suit the goal in a particular domain and developing procedural recognized by constructing the dynamic models of the periodic pattern of human movements and are highly dependent on the robustness of the tracking.

Spatio-temporal relations are the basis for many of the selections users perform when they formulate queries for the purpose of semantic-based motion retrieval. Although such query languages use natural-language-like terms, the formal definitions of these relations rarely reflect the language people would use when communicating with each other. To bridge the gap between the computational models used for spatio-temporal relations and people's use of motion verbs in their natural language, a model of these spatio-temporal relations was calibrated for motion verbs.

In the previous works, the retrieval using spatio-temporal relations is similar trajectory retrieval, it's only the content-based retrieval but not semantic-based. Therefore, we propose a novel approach for motion recognition from the aspect of semantic meaning in this paper. This issue can be addressed through a hierarchical model that explains how the human language interacts with motions and we evaluate our new approach using trajectory distance based on spatial relations to distinguish the conceptual similarity and get the satisfactory results. In the experiment and application part, we apply the proposed approach to semantic recognition of motions and trajectory retrieval. Extending our novel motion verbs model with more abundant motion verbs for gapping the chasm between high-level semantics and low-level video feature is our further consideration. Finally, we can apply a security using CCTV through this study.

\section{Related Works}

The trajectory of a moving object is a presentation of spatio-temporal relationship between moving objects and an important element in video indexing for contentbased retrieval. In Temporal relation, John Z. Li et al.[14] represented the trajectory of a moving object as eight directions - North(NT), Northwest(NW), Northeast(NE), West(WT), Southwest(SW), East(ET), Southeast(SE) and Southwest(SW). There are many researches on spatial relations of moving objects. Martin Erwig[2] is defined spatio- temporal predicates and Our previous work[3] was proposed Hierarchical relation modeling about moving object. Lee[4][13] have proposed relations such as $3 \mathrm{D} C$-string for the various extensions of the original representations of $2 \mathrm{D}$ and $\mathrm{W}$ Ren, M Singh and S Singh[7] proposed to combine directional relations with topological relations by specifying six spatial relationships: 'Left', 'Right', 'Up', 'Down', 'Touch', and 'Front'. Pei-Yi Chen[8] measure velocity similarity by six possible velocity trends. The SMR scheme by Chang et al.[5][6] makes use of fifteen topological operators to describe the spatial relationships between moving object and non-moving object and also makes a new scheme to identify objects. Egnehofer and Franzosa[1] that proposed eight topological spatial relationships including 'equal', 'inside', 'cover', 'overlap', 'touch', 'disjoint', 'covered-by' and 'contains'. This model is the most famous study. 


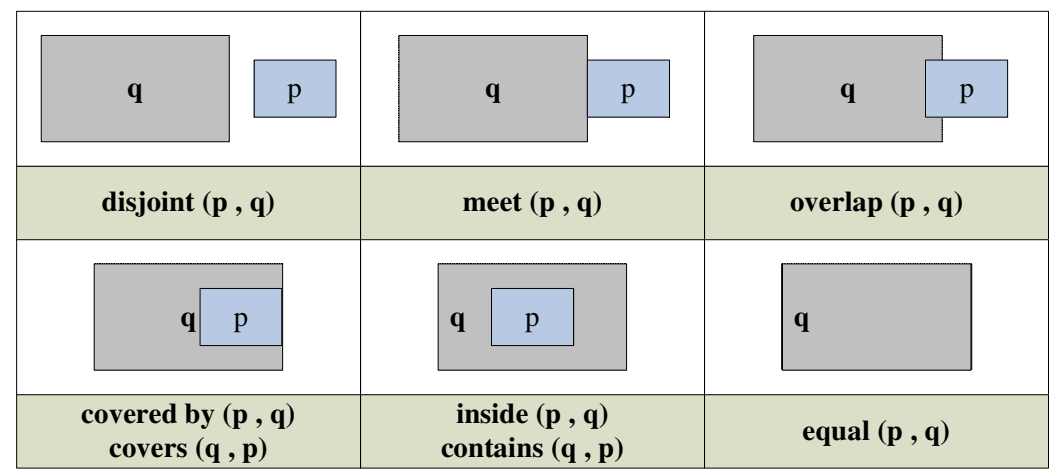

Fig. 1. Topological Relation of Egnehofer and Franzosa

For example, if two regions are disjoint and later they overlap, then there must have been a topological situation where both regions have met each other and they cannot move directly such as, from disjoint to equal in the fig. 2 .

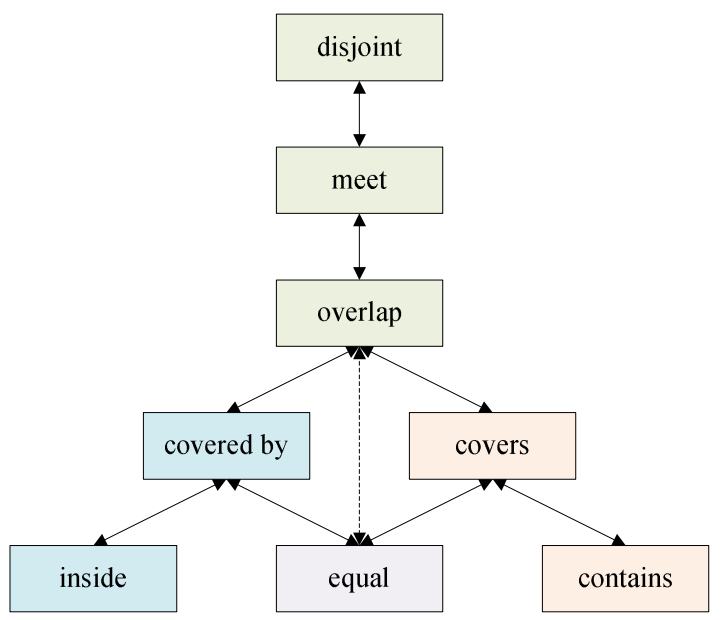

Fig. 2. Conceptual neighborhood graph

However, Most of study represents motion between 2 moving objects and some study is not sufficient to provide a complete representation of the semantic motion based on directional relations by ignoring topological relations and describing the concept or semantics motion. Therefore, we represent the semantics between moving object and non-moving object through topological relations that we make elements of motion. We also match motion verbs so that they don't have direction features for intrusion detection using CCTV[15][16]. 


\section{Previous Work}

In the previous work[3], we propose a novel approach for motion recognition from the aspect of semantic meaning. We define 5 basic elements to represent spatial relations between non-moving object and moving object, and then expand them through a hierarchical model. Therefore, it specifies how to give definitions to the motions of general moving objects by the spatio-temporal relation, and match between the definition of moving objects and motion verbs in the vocabulary.

The fig. 3 shows hierarchical semantic relations[11] for motion verbs, in other words, motion verbs and visual information map from low-level features to the semantic-level. Our model is divided into 3 levels. The level 1 describes basic elements of motion, level 2 is the extension of basic elements and level 3 shows combination of level 2. And, we represent semantic relation between motions and motion verbs for each element. For example, 'go into' and 'go out' are subclasses of motion word 'go through' which was set with 'Part_of' relation and 'go_to'('come up) can be inserted into 'go_through', it also has a relation of antonym between 'go_out'(depart) and 'enter'. Even if each motion verb is different in directional and spatial relations, the semantic relation is the same.

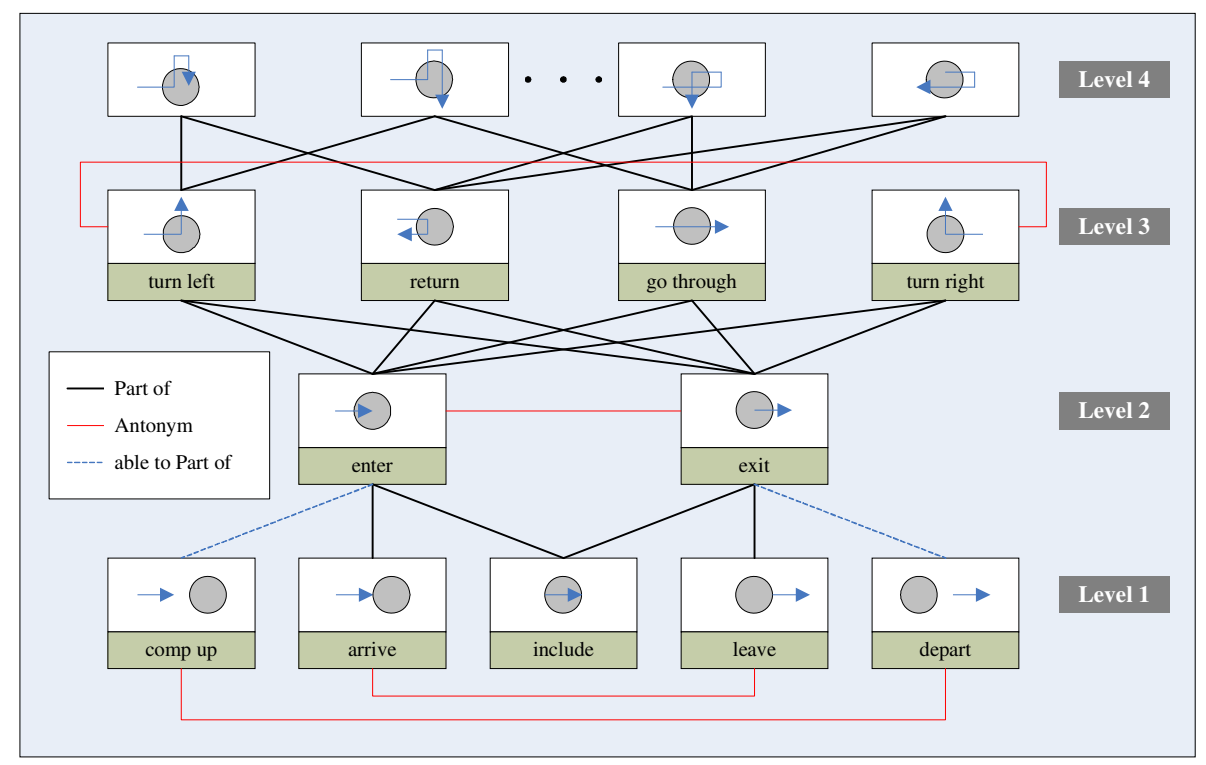

Fig. 3. Hierarchical relation modeling about moving object

However, it's difficult to distinguish semantics only using topological relations in level 3, which contains trajectories in the same topological relation. That is, we have to consider directional relations. We introduce proposed new spatio-temporal relation in next section and measure trajectory similarity using TSR which is represented moving distance and angle. 


\section{Building of Extended Spatio-temporal Relation}

In this section we divided non-directional relation and directional relation.

\subsection{Modeling of Non-directional Relation Motion}

In the fig. 3 is cannot representation of all of Motion so we extended our spatiotemporal relation using Egenhofer's model and our previous work. Firstly, we define motion using initial and final topological relation. For example, if initial topological relation is Disjoint and Final topological relation is Disjoint or Meet fallowing fig. 2, table 1 is shown motion. For the motion, we considered the number of all cases.

Table 1. Representation of Topological Relation about disjoint

\begin{tabular}{cccccc}
\hline \multirow{2}{*}{ No. } & \multicolumn{2}{c}{ Initial } & \multicolumn{2}{c}{ Final } & Motion \\
\hline 1 & Topology & position & Topology & position & disjoint \\
2 & 1 & disjoint & 1 & go to \\
3 & disjoint & 1 & disjoint & 1 & surround \\
4 & disjoint & 1 & disjoint & 1 & go by \\
5 & disjoint & 1 & meet & 1 & arrive \\
6 & disjoint & 1 & disjoint & 2 & around \\
& disjoint & 2 & disjoint & 2 & depart \\
& disjoint & 1 & overlap & 1 & X \\
& disjoint & 1 & covered by & 1 & X \\
& disjoint & 1 & covers & 1 & X \\
& disjoint & 1 & inside & all & X \\
& disjoint & 1 & contains & 1 & X \\
\hline
\end{tabular}
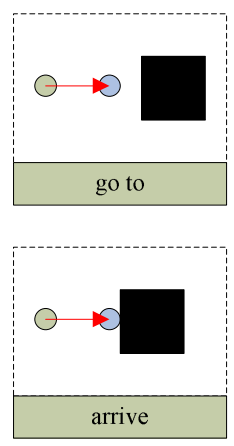
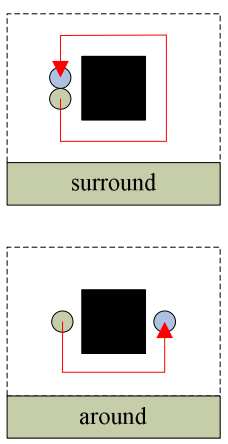
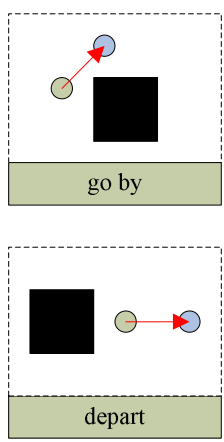

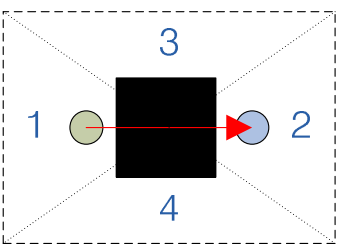

Fig. 4. Motion and Topological Position

Fig. 4 is shown motion and motion verbs also the position is shown in the table 1 . Actually, Disjoint is can move the disjoint and meet but it cannot move the others such as overlap, inside and etc. from fig. 2. And disjoint can represent go to, surround, go by, arrive, around and depart from initial and final position. Table 2 is the representation of topological relation about others. 
Table 2. Representation of Topological Relation about others

\begin{tabular}{cccccc}
\hline \multirow{2}{*}{ No. } & Topology & position & Topology & position & Motion \\
\hline 7 & meet & 1 & disjoint & 1 & leave \\
8 & meet & 1 & meet & 1 & contact \\
9 & meet & 1 & overlap & 1 & enter \\
10 & overlap & 1 & meet & 1 & exit \\
11 & overlap & 1 & overlap & 1 & contact \\
12 & overlap & 1 & covered by & 1 & enter \\
13 & overlap & 1 & covers & 1 & include \\
14 & overlap & 1 & equal & 1 & same \\
15 & covered by & 1 & overlap & 1 & exit \\
16 & covered by & 1 & covered by & 1 & included by \\
17 & covered by & 1 & inside & all & included by \\
18 & covers & 1 & overlap & 1 & exit \\
19 & covers & 1 & covers & 1 & include \\
20 & covers & 1 & contains & 1 & include \\
21 & inside & all & covered by & all & included by \\
22 & inside & all & inside & all & included by \\
23 & contains & all & covers & all & include \\
24 & contains & all & contains & all & include \\
25 & equal & - & overlap & all & exit \\
26 & equal & - & equal & - & same \\
\hline
\end{tabular}

We get 14 motions from table 1 and 2 . Some motion verb is including several motions. Even if initial position changed, it is not a problem because we consider all of one way direction. This means if initial position changed, final position also changed. Other case is combination of motion we can solve using combination and it made a new model such as fig. 5. For example DJ_OL from table 3(For example, if initial topological relation is DJ and Final topological relation is also DJ, we'll represent DJ_DJ) is can be representation using fig. 2 but we need this case so we combine DJ_ME with ME_OL. In this case 2DJ is a sufficient condition for DJ_OL.

Table 3. Representation of Topological Relation

\begin{tabular}{cc||cc}
\hline Representation & Topological Relation & Representation & Topological Relation \\
\hline DJ & Disjoint & CD & Covered by \\
ME & Meet & CR & Covers \\
OL & Overlap & IN & Inside \\
EQ & Equal & CN & Contains \\
\hline
\end{tabular}

Finally, we define 51 motions it doesn't consider object size. It means that some motion represent covered by or covers between moving object size and non-moving object size so except this case we get 51 motions. 


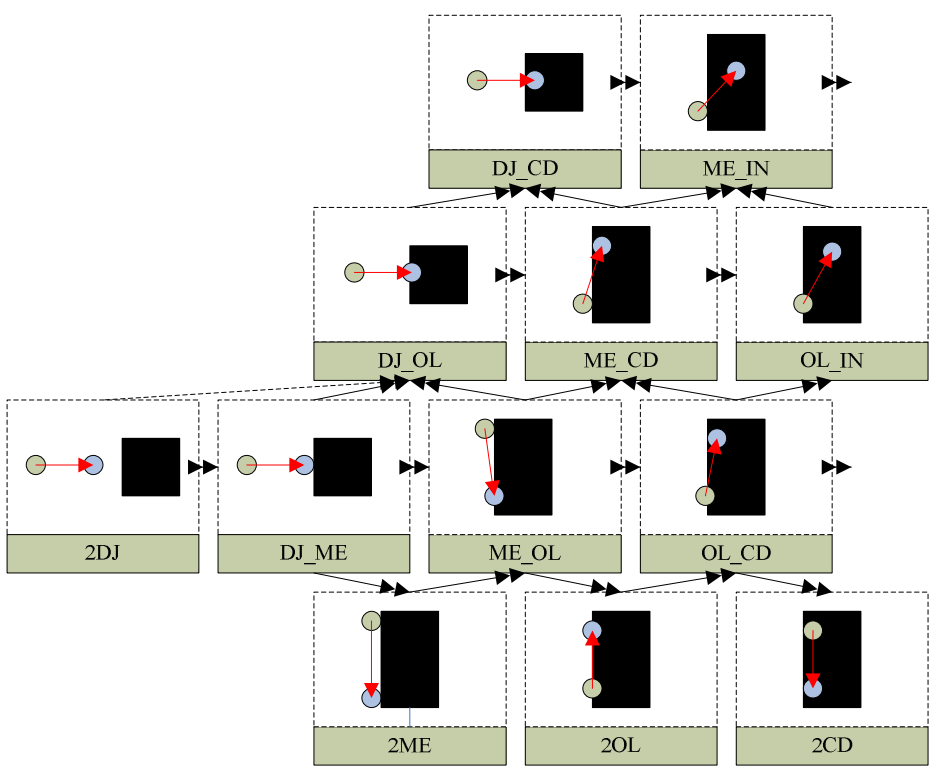

Fig. 5. Part of Proposed Motions

\subsection{Modeling of Directional Relation Motions}

Some case gets a directional relation such as table 4 and fig. 6 . They represent a different motion following final position. In this paper, we just consider 4 directions such as fig. 4.

Table 4. Topological Relation including Directional Relation

\begin{tabular}{cccccc}
\hline \multirow{2}{*}{ No. } & \multicolumn{2}{c}{ Initial } & \multicolumn{2}{c}{ Final } & \multirow{2}{*}{ Motion } \\
& Topology & position & Topology & position & return \\
1 & disjoint & 1 & disjoint & 1 & go through \\
2 & disjoint & 1 & disjoint & 2 & turn left \\
3 & disjoint & 1 & disjoint & 3 & turn right \\
4 & disjoint & 1 & disjoint & 4 & \\
\hline
\end{tabular}
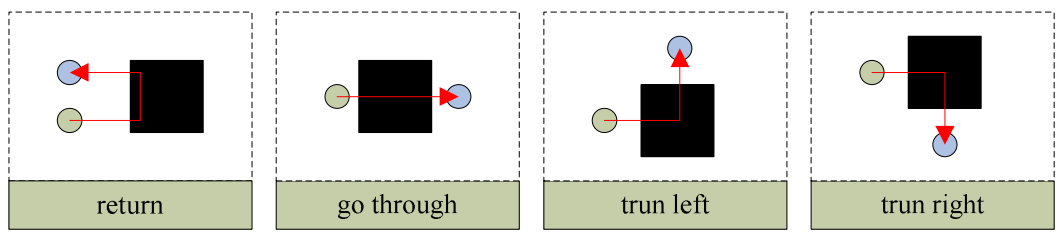

Fig. 6. Motions including Directional Relation 


\section{Similarity Measure between Motions}

In this section, we introduce the method of similarity measure non-directional relation and directional relation. Similarity Measure Non-Directional Relation model is using our proposed section 4 and Directional Relation model is using TSR(Tangent Space Representation).

\subsection{Similarity Measure Non-directional Relation Model}

We proposed spatio-temporal relation in previous section. This relation is continuously moving of object following temporal relation. In fig 7 , similarity (distance) is 9 from DJ1_DJ1 (number is position of table 1 and 2) to DJ2_DJ2 and it is 10 from DJ1_DJ1 to go through(directional relation). We can get a similarity between moving objects. For example similarity is 5 from CD1_IN to CD2_OL2. Another case, similarity is 3 from DJ1-DJ1 to ME1_IN.

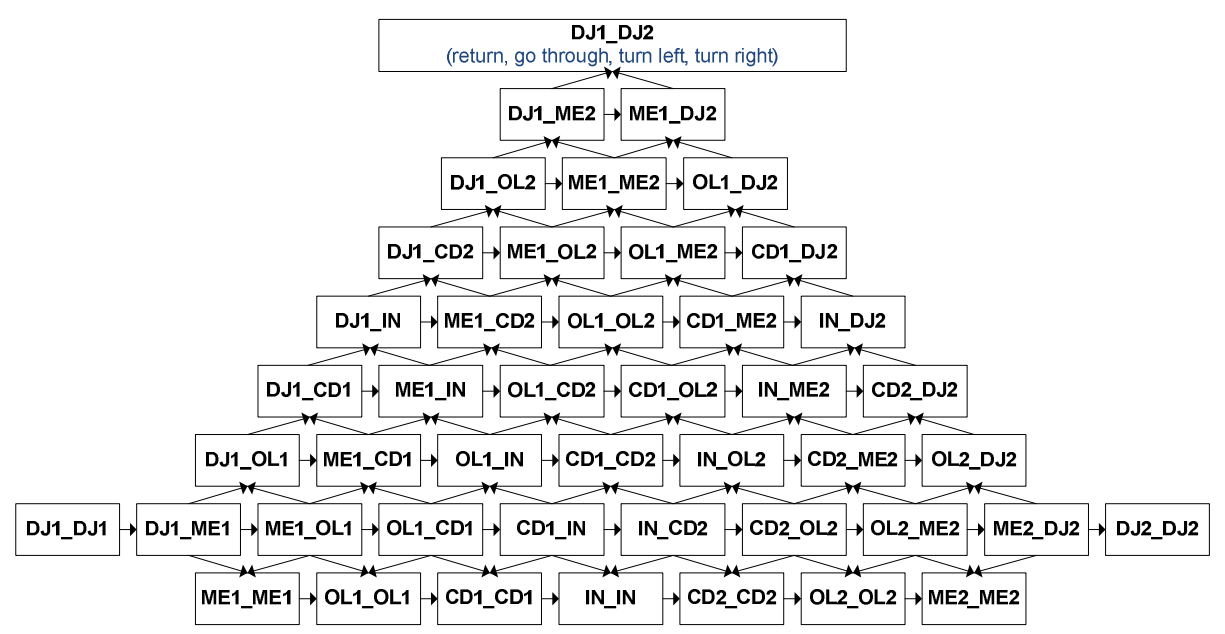

Fig. 7. Proposed Spatio-temporal Relation

For the similarity, we are using the LCM (Least Common Multiple). For example, let's get a similarity between ME1_IN and DJ1_DJ1. ME1_IN can be divided ME1_CD1 and OL1_IN. Also ME1_CD1 is can be divided ME1_OL1 and OL1_CD1 such as fig. 8. OL1_IN is a union between OL1_CD1 and CD1_IN. DJ1_DJ1is a basic element and cannot divide. Using this, we can get a 3 distance $-2,3$ and 4 (between ME1_OL1 and DJ1_DJ1, between OL1_CD1 and DJ1_DJ1, between CD1_IN and DJ1_DJ1). Therefore similarity is $1 / 3(2+3+4)=3$.

$$
\begin{gathered}
\text { ME1_IN = ME1_OL1 + OL1_CD1+ CD1_IN } \\
\text { DJ1_DJ1 = DJ1_DJ1 + DJ1_DJ1 + DJ1_DJ1 } \\
\text { Similarity }=1 / 3(2+3+4)=3
\end{gathered}
$$




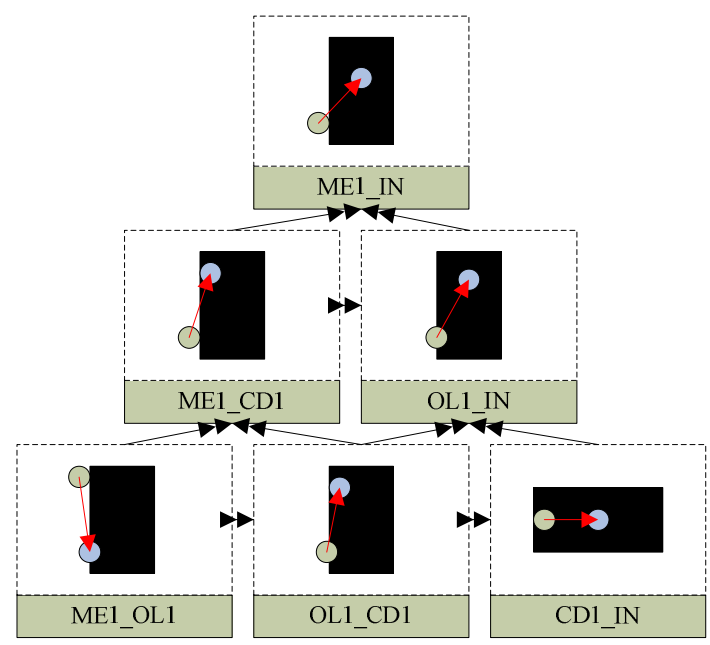

Fig. 8. Proposed Spatio-temporal Relation

\subsection{Similarity Measure Directional Relation Model}

TSR(Tangent Space Representation)[9][10] is a kind of method to measure similarity about a object's shape in two or three dimensions. Actually, polygonal shapes consist of lines and the line also contains points, so we used TSR for measuring similarity between trajectories. In the figure 9, Object $A$ and Object $B$ is a same shape. The right graph is TSR about Object $A$ and Object $B$. The abscissa is sum of distance about lines and the ordinate is sum of angle between lines. In this graph, there isn't difference about area so they are same shape. In this way, we applied to measure similarity between trajectories using TSR.

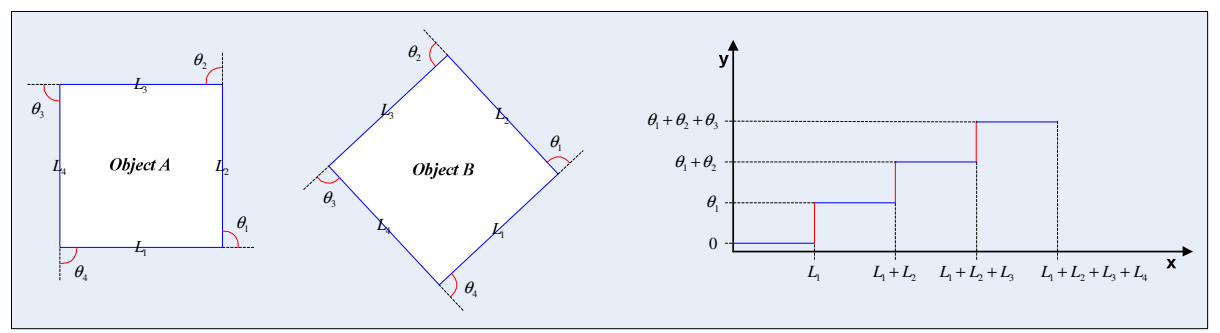

Fig. 9. Similarity Measure Methods of Object Shape

In the figure 10, the left part shows sample motions about 'turn_left'. The Rusult(table 5) is that they are the same semantic so the right figure shows the same graph about a group of 'turn_left'. We get the value after measuring similarity between trajectories. In this study, if two trajectories are the same, the similarity between those is 0 . In other words, similarity is in inverse proportion to measured value. The sample trajectory is the most similar with 'turn left' in table 5. 


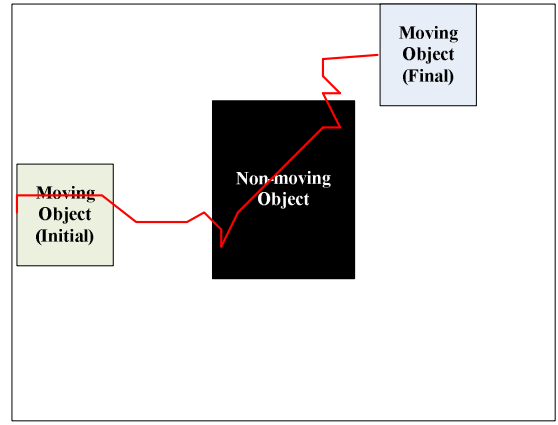

Fig. 10. Sample trajectory of moving object

Table 5. Similarity Measure using sample trajectory

\begin{tabular}{cccc}
\hline & go_through & turn_left & return \\
\hline Similarity & 51.26031 & 30.4637 & 97.0059 \\
\hline
\end{tabular}

\section{Experiment}

We experiment on semantic-based trajectory retrieval with the proposed method. For the experiment, we make 100 coordinate set (non-moving object's coordinate set and moving object's trajectory) using simulator. The result is $97 \%$ (3\% is simulator error) because is not a real video data. This paper is a just proposed Extended Spatiotemporal relation so we did use a real video data because problem of object detection and trajectory extraction. Therefore the result doesn't have a any meaning. It just checks our model. In previous work[3], we got a $92.857 \%$ using previous model + TSR and when we use proposed model, the precision rate increase to almost $100 \%$.

Difference between previous work and this work as following :

A. Number of models (before: 6, now: 51) - except directional relation and size of moving object. Therefore, previous model cannot represent all of case but this work considered the number of all cases.

B. Max distance is just 4 in previous work and it is 10 in this work so we can get similarity of various models in non-directional relation.

C. This research is inserted initial and final position so it's better than before work for the retrieval of motions such as intrusion detection using CCTV.

\section{Conclusion and Future Works}

In this paper, we proposed new spatio-temporal relation. Previous work, we cannot representation all of models but we considered the number of all cases in this study. 
Increasing number of models, we limit a mapping between motions and motion verbs so we define motion verb about the 14 basic model and others are representation using topological relation and position. We be sure when applying intrusion detection system, get a good result therefore next study we will apply this using real video data and CCTV.

Acknowledgments. This work was supported by the Korea Science and Engineering Foundation (KOSEF) grant funded by the Korea government (MEST) (No. 20090064749) and by the National Research Foundation of Korea (NRF) grant funded by the Korea government (MEST) (No. 2010-0011656).

\section{References}

1. Egenhofer, M., Franzosa, R.: Point-set topological spatial relations. International Journal of Geographical Information Systems 5(2), 161-174 (1991)

2. Erwig, M., Schneider, M.: Spatio-Temporal Predicates. IEEE TKDE 14(4), 881-901 (2002)

3. Cho, M., Choi, C., Choi, J., Yi, H., Kim, P.-K.: Trajectory Annotation and Retrieval Based on Semantics. In: Boujemaa, N., Detyniecki, M., Nürnberger, A. (eds.) AMR 2007. LNCS, vol. 4918, pp. 251-264. Springer, Heidelberg (2008)

4. Lee, A.J.T., Chiu, H.-P., Yu, P.: Similarity Retrieval of Videos by using 3D C-String knowledge Representation. Journal of Visual Communication \& Image Representation, 749-773 (2005)

5. Chang, J.W., Kim, Y.j., Chang, K.J.: A Spatial Match Representation Scheme for Indexing and Querying in Iconic Image Databases. In: ACM International Conference on Information and Knowledge Management, pp. 169-176 (November 1997)

6. Chang, J.-W., Kim, Y.-J.: Spatial-Match Iconic Image Retrieval with Ranking in Multimedia Databases. In: Proceedings of Advances in Web-Age Information Management: Second International Conference (July 2001)

7. Ren, W., Singh, M., Singh, S.: Image Retrieval using Spatial Context. In: 9th International Workshop on Systems, Signals and Image Processing (November 2002)

8. Chen, P.-Y., Chen, A.L.P.: Video Retrieval Based on Video Motion Tracks of Moving Objects. In: Proceedings of SPIE, vol. 5307, pp. 550-558 (2003)

9. Baek, S., Hwang, M., Cho, M., Choi, C., Kim, P.: Object Retrieval by Query with Sensibility based on the Kansei-Vocabulary Scale. In: Huang, T.S., Sebe, N., Lew, M., Pavlović, V., Kölsch, M., Galata, A., Kisačanin, B. (eds.) ECCV 2006 Workshop on HCI. LNCS, vol. 3979, pp. 109-119. Springer, Heidelberg (2006)

10. Hwang, M., Baek, S., Kong, H., Shin, J., Kim, W., Kim, S., Kim, P.: Adaptive-Tangent Space Representation for Image Retrieval based on Kansei. In: Gelbukh, A., Reyes-Garcia, C.A. (eds.) MICAI 2006. LNCS (LNAI), vol. 4293, pp. 828-837. Springer, Heidelberg (2006)

11. Cho, M., Song, D., Choi, C., Choi, J., Park, J., Kim, P.-K.: Comparison between Motion Verbs using Similarity Measure for the Semantic Representation of Moving Object. In: Sundaram, H., Naphade, M., Smith, J.R., Rui, Y. (eds.) CIVR 2006. LNCS, vol. 4071, pp. 281-290. Springer, Heidelberg (2006)

12. Aghbari, Z.A.: Studies on Modeling and Querying Video Databases. Degree of Doctorate of Philosophy, Kyushu University (2001) 
13. Lee, S.Y., Hsu, F.J.: Spatial reasoning and similarity retrieval of images using 2D C-String knowledge representation. Pattern Recognition 25(3), 305-318 (1992)

14. Li, J.Z., Ozsu, M.T., Szafron, D.: Modeling of Moving Objects in a Video Data-base. In: Proceedings of the International Conference on Multimedia Computing and Systems, pp. 336-343 (1997)

15. Pieters, W.: Representing Humans in System Security Models: An Actor-Network Approach. Journal of Wireless Mobile Networks, Ubiquitous Computing, and Dependable Applications 2(1), 75-92 (2011)

16. Sun, J., Wang, Y., Si, H., Yuan, J., Shan, X.: Aggregate Human Mobility Modeling Using Principal Component Analysis. Journal of Wireless Mobile Networks, Ubiquitous Computing, and Dependable Applications q(2/3), 83-95 (2010) 